\title{
THE THIRD COHOMOLOGY GROUP OF A RING AND THE COMMUTATIVE COHOMOLOGY THEORY
}

\author{
BY MURRAY GERSTENHABER ${ }^{1}$
}

Communicated April 8, 1967

The cohomology groups of a ring depend not only on the ring but on a choice of category of which the ring is a member. In [4] it was shown that under very weak conditions on the category one could define the third cohomology group $\varepsilon^{3}(A, M)$ of a ring $A$ with coefficients in a bimodule $M$ as certain equivalence classes of exact sequences

$$
0 \rightarrow M \rightarrow N \stackrel{\rho}{\rightarrow} B \rightarrow A \rightarrow 0 .
$$

The groups $\mathcal{E}^{1}(A, M)$ and $\mathcal{E}^{2}(A, M)$ were the derivations of $A$ into $M$ and extensions of $A$ by $M$, respectively. We show here that if $\mathfrak{a}$ is an ideal of $A$ and if $M$ is an $A / \mathfrak{a}$ module, then there is an exact sequence

$$
\begin{aligned}
& 0 \rightarrow \mathcal{E}^{1}(A / \mathfrak{a}, M) \stackrel{j_{1}^{*}}{\rightarrow} \varepsilon^{1}(A, M) \stackrel{i_{1}^{*}}{\rightarrow} \operatorname{Hom}_{A}(\mathfrak{a}, M) \stackrel{\Delta_{1}}{\rightarrow} \\
& \varepsilon^{2}(A / \mathfrak{a}, M) \stackrel{j_{2}^{*}}{\rightarrow} \varepsilon^{2}(A, M) \stackrel{i_{2}^{*}}{\rightarrow} \mathrm{e} \stackrel{\Delta_{2}}{\rightarrow} \varepsilon^{3}(A / \mathfrak{a}, M) \stackrel{j_{3}^{*}}{\rightarrow} \varepsilon^{3}(A, M),
\end{aligned}
$$

where $\mathcal{C}$ is an explicitly described submodule of $\operatorname{Ext}_{A}^{1}(\mathfrak{a}, M)$. (Cf. Harrison [5, Theorem 2].) We then show that for the category of commutative associative algebras over a coefficient field $k$, the group $\varepsilon^{3}(A, M)$ as defined in [4] coincides with that defined by Harrison in [5]. (An example of Barr in a note to appear [1] shows that in the category of commutative associative algebras, $\varepsilon^{3}(A, M)$ is not the first derived functor of the Baer group $\varepsilon^{2}(A, M)$ when the latter is considered as a functor of the module $M$.) More generally, two cohomology theories for a category of algebras or groups with sufficiently many projectives coincides if (i) each possesses an exact sequence analogous to (2) with $\varepsilon^{1}(A, M)$ the derivations of $A$ into $M$, and (ii) $\varepsilon^{n}(A, M)=0$ whenever $A$ is projective.

In order to be brief, we prove the exactness of (2) explicitly only for commutative associative algebras over $k$, but the reader of [4] will observe that the considerations apply to any "category of interest" in the sense of that paper.

1 The author gratefully wishes to acknowledge the support of the Institute for Advanced Study and of the N.S.F. through Grant GP 3683 with the University of Pennsylvania. 
1. The exactness of the long sequence. The category being commutative algebras over $k$, an " $A$-module $M$ " is a bimodule with $a m=m a$ for all $a \in A, m \in M$. The group $\mathcal{e}$ consists of the equivalence classes of $A$-module extensions

$$
0 \rightarrow M \rightarrow N \stackrel{\sigma}{\rightarrow} \mathfrak{a} \rightarrow 0
$$

such that $\sigma(n) n^{\prime}=n \sigma\left(n^{\prime}\right)$ for all $n n^{\prime} \in N$. We make $N$ into an algebra by setting $n n^{\prime}=\sigma(n) n^{\prime}$. The definition and the exactness of the long sequence (2) are classical until one gets to $\mathcal{C}$. Recall that $\mathcal{E}^{3}(A, M)$ is the group of equivalence classes of exact sequences (1) in which (1) $B$ and $N$ are commutative $k$-algebras, $N$ is a $B$-module, and $\rho$ is a $B$-module morphism with $\rho(n) n^{\prime}=n n^{\prime}=n \rho\left(n^{\prime}\right)$ for all $n, n^{\prime} \in N$, and (2) $B \rightarrow A$ is a ring morphism and $M \rightarrow N$ is a $B$-module morphism, where $M$ is a $B$-module by virtue of the morphism $B \rightarrow A$.

We have

$$
0 \rightarrow \mathfrak{a} \stackrel{i}{\rightarrow} A \stackrel{j}{\rightarrow} A / \mathfrak{a} \rightarrow 0,
$$

$M$ is an $A$-module by virtue of the morphism $j$, and $\mathfrak{a} M=M \mathfrak{a}=0$. If

$$
E: 0 \rightarrow M \rightarrow B \stackrel{\pi}{\rightarrow} A \rightarrow 0
$$

represents an element of $\varepsilon^{2}(A, M)$ then it is trivial to verify that the element $i_{1}^{*} E$ of $\operatorname{Ext}_{A}^{1}(\mathfrak{a}, M)$ represented by $0 \rightarrow M \rightarrow \pi^{-1}(\mathfrak{a}) \rightarrow \mathfrak{a} \rightarrow 0$ lies in C. If $i_{1}^{*} E$ splits by a map $s: \mathfrak{a} \rightarrow \pi^{-1}(\mathfrak{a}) \subset B$, then $s a$ is an ideal of $B$ and $0 \rightarrow M \rightarrow B / s \mathfrak{a} \rightarrow A / \mathfrak{a} \rightarrow 0$ represents an element of $\varepsilon^{2}(A / \mathfrak{a}, M)$ whose image under $j_{1}^{*}$ is $E$. The exactness of (2) at $\varepsilon^{2}(A, M)$ follows.

Let

$$
F: 0 \rightarrow M \rightarrow N \stackrel{\sigma}{\rightarrow} \mathfrak{a} \rightarrow 0
$$

represent an element of $\mathcal{C}$ and set $\rho=i \sigma$. Then

$$
0 \rightarrow M \rightarrow N \stackrel{\rho}{\rightarrow} A \rightarrow A / \mathfrak{a} \rightarrow 0
$$

by definition represents $\Delta_{2} F \in \mathcal{E}^{3}(A / \mathfrak{a}, M)$. If $\Delta_{1} F=0$, then by Theorem 4 of [4] there is a commutative diagram

$$
\begin{aligned}
& 0 \rightarrow N \rightarrow B \rightarrow A / \mathfrak{a} \rightarrow 0 \\
& \quad \downarrow \quad \| \\
& 0 \rightarrow M \rightarrow N \rightarrow A \rightarrow A / \mathfrak{a} \rightarrow 0
\end{aligned}
$$

and hence an extension $E: 0 \rightarrow M \rightarrow B \rightarrow A \rightarrow 0$ of which one may verify that $i_{2}^{*} E=F$. The exactness of (2) at $\mathbb{e}$ follows. The fact that 
(2) is at least a zero sequence at $\varepsilon^{3}(A / \mathfrak{a}, M)$ is trivial leaving us only to prove that if

$$
E: 0 \rightarrow M \rightarrow N \stackrel{\rho}{\rightarrow} B \rightarrow A / \mathfrak{a} \rightarrow 0
$$

has the property that $j^{*} E=0$ then $E$ is of the form $\Delta_{2} F$. (3) If $j^{*} E=0$, then using Theorem 4 of [4] we have a commutative diagram

$$
\begin{aligned}
& 0 \quad 0 \\
& F: 0 \rightarrow M \rightarrow C \stackrel{\theta^{\dagger}}{\rightarrow} \mathfrak{a} \rightarrow 0 \\
& \downarrow \quad \downarrow \quad \downarrow \\
& 0 \rightarrow N \rightarrow \bar{B} \rightarrow A \rightarrow 0 \\
& E: 0 \rightarrow M \rightarrow N \rightarrow B \rightarrow A / \mathfrak{a} \rightarrow 0 \\
& \downarrow \quad \downarrow
\end{aligned}
$$

It is easy to see that $(\theta c) c^{\prime}=c c^{\prime}=c\left(\theta c^{\prime}\right)$ for all $c, c^{\prime} \in C$, and that if $N$ and $C$ are considered as ideals in $\bar{B}$ then $N C=C N=0$; therefore $C$ becomes an $A$-module by setting $a c=b c$ where $b$ is any element of $\bar{B}$ projecting onto $a$. Thus $F$ represents an element of $\mathfrak{e}$ and it remains only to show that $\Delta_{2} F$ is equivalent to $E$. Now let $C+N$ denote the sum of $C$ and $N$ in $\bar{B}$ and observe that $C \cap N=M$, whence defining $C \oplus N \rightarrow C+N$ by $(c, n) \rightarrow c-n$ we have a short exact sequence $0 \rightarrow M \rightarrow C \oplus N \rightarrow C+N \rightarrow 0$. Since $C$ and $N$ are both ideals in $\bar{B}$ it follows that $C \oplus N$ is a $B$-module in an obvious way. Moreover, the kernel of the composite morphism $\bar{B} \rightarrow A / \mathfrak{a}$ in (3) is just $C+N$, so we have a composite sequence $E: 0 \rightarrow M \rightarrow C \oplus N \rightarrow \bar{B} \rightarrow A / \mathfrak{a} \rightarrow 0$ representing an element of $\varepsilon^{3}(A, M)$. But we have the obvious morphisms

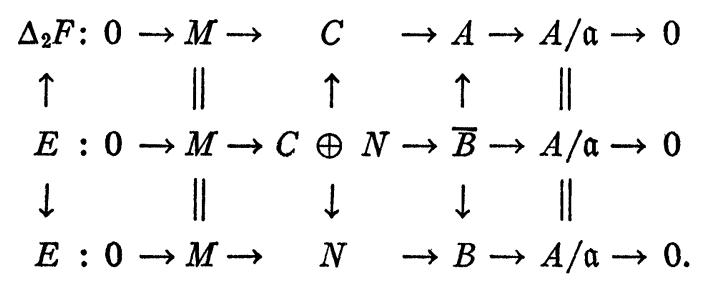

Thus $E$ and $\Delta_{2} F$ are equivalent, proving the exactness of the long sequence (2).

We have not required that the coefficient ring be a field nor have we used any special property of the commutative theory. Observe, 
however, that if, as we suppose, the category is that of commutative rings, $M$ is an $A / \mathfrak{a}$ module, and

$$
0 \rightarrow M \rightarrow N \stackrel{\sigma}{\rightarrow} \mathfrak{a} \rightarrow 0
$$

is an additively split sequence of $A$-modules, with $\sigma(n) n^{\prime}=n \sigma\left(n^{\prime}\right)$, all $n, n^{\prime} \in N$, then choosing a splitting map $s: \mathfrak{a} \rightarrow N$ represents $N$ as the group direct sum $a+M$ with $a(x, m)=(a x, a m+F(a, x))=(x, m) a$, where $a \in A, x \in \mathfrak{a}, m \in M$, and $F$ is a biadditive map $A \times M \rightarrow M$. Since $\sigma(x, m)=x$ and $\mathfrak{a} M=0$, we have $\sigma(x, m) \cdot\left(x^{\prime}, m^{\prime}\right)=\left(x x^{\prime}, F\left(x, x^{\prime}\right)\right)$ and the condition that $\sigma(n) n^{\prime}=n \sigma\left(n^{\prime}\right)$ then readily implies that $F\left(x, x^{\prime}\right)=F\left(x^{\prime}, x\right)$. Setting $F(x, a)=F(a, x), F$ becomes a symmetric map $[A \otimes M+M \otimes A] \rightarrow M$. From $(a b)(x, m)=a[b(x, m)]$ we have $a F(b, x)-F(a b, x)+F(a, b x)=0$. Since $F$ has its values in $M$ we set $F(a, b) x=0$ even though $F(a, b)$ is undefined, and have thus $\delta F(a, b, x)=0$, where $\delta$ is the Hochschild coboundary [6]. It is trivial to verify that $\delta F(a, b, c)=0$ whenever $a, b$ or $c$ is in $a$, that changing the splitting replaces $F$ by $F+\delta g$ where $g$ is a linear map $a \rightarrow M$, and that $\mathfrak{C}$ is naturally isomorphic to the quotient,

(symmetric cocycles)/(coboundaries).

2. Harrison's sequence. All algebras and modules are now assumed to be vector spaces over a field $k$. For every $i$, set $A^{(i)}=\otimes^{i} A$ and set $V^{(n)}=\sum_{i=0}^{n-1} A^{(i)} \otimes \mathfrak{a} \otimes A^{(n-i-1)} \subset A^{(n)}$. We have the exact sequence

$$
0 \rightarrow V^{(n)} \stackrel{i_{n}}{\rightarrow} A^{(n)} \stackrel{j_{n}}{\rightarrow}(A / \mathfrak{a})^{(n)} \rightarrow 0 .
$$

Let $C^{n}(A, M)$ denote the submodule of $\operatorname{Hom}_{k}\left(A^{(n)}, M\right)$ consisting of all elements vanishing on "shuffles" (cf. [3]), define $C^{n}(A / \mathfrak{a}, M)$ similarly, and let $C^{n}(V, M)$ denote the set of those elements of $\operatorname{Hom}_{k}\left(V^{(n)}, M\right)$ which vanish on shuffles in which one of the elements shuffled is in $\mathfrak{a}$. Since $\mathfrak{a} M=M \mathfrak{a}=0$, if $F \in C^{n}(V, M)$ then $\delta F$ is a welldefined element of $C^{n+1}(V, M)$. We have, thus, $\delta_{A}^{n}: C^{n}(A, M)$ $\rightarrow C^{n+1}(A, M)$, similarly with $A / \mathfrak{a}$ in place of $A$, and $\delta_{V}^{n}: C^{n}(V, M)$ $\rightarrow C^{n+1}(V, M)$. Harrison sets $\varepsilon^{n}(A, M)=\operatorname{ker} \delta_{A}^{n} /$ im $\delta_{A}^{n-1}$, and similarly for $A / \mathfrak{a}$. Note that $\operatorname{im} \delta^{0}=0$ and that $\operatorname{ker} \delta_{V}^{1}=\operatorname{Hom}_{A}(\mathfrak{a}, M)$. To be consistent with Harrison's notation we set ker $\delta_{V}^{n} /$ im $\delta_{V}^{n-1}$ $=\mathfrak{e}^{n-1}(A, \mathfrak{a}, M)=\mathfrak{e}^{n-1}$. Define $\Delta: \quad \mathfrak{e}^{n-1} \rightarrow \mathcal{E}^{n+1}(A / \mathfrak{a}, \quad M)$ so: if $F \in$ ker $\delta_{V}^{n}$ let $\bar{F}$ be any element of $\mathfrak{C}^{n}(A, M)$ such that $i_{n}^{*} \bar{F}=F$. Then $\delta \bar{F}$ vanishes on $V^{(n+1)}$ and so may be viewed as an element of $\operatorname{ker} \delta_{A / \mathfrak{a}}^{n+1}$ whose cohomology class $\Delta F$ in fact depends only on the class of $F$. It is not difficult to verify that we then have a long exact sequence 


$$
\begin{aligned}
0 & \rightarrow \mathcal{E}^{1}(A / \mathfrak{a}, M) \stackrel{i_{1}^{*}}{\rightarrow} \mathcal{E}^{1}(A, M) \stackrel{j_{1}^{*}}{\rightarrow} \operatorname{Hom}_{A}(\mathfrak{a}, M) \stackrel{\Delta}{\rightarrow} \mathcal{E}^{2}(A / \mathfrak{a}, M) \\
& \stackrel{i_{2}^{*}}{\rightarrow} \cdots \rightarrow \mathcal{E}^{n}(A / \mathfrak{a}, M) \stackrel{i_{n}^{*}}{\rightarrow} \mathcal{E}^{n}(A, M) \stackrel{j_{n}^{*}}{\rightarrow} \mathbb{C}^{n-1}(A, \mathfrak{a}, M) \\
& \stackrel{\Delta}{\rightarrow} \mathcal{E}^{n+1}(A / \mathfrak{a}, M) \rightarrow \cdots
\end{aligned}
$$

Now the "symmetric cocycles" of $\$ 1$ are simply the elements of $\operatorname{ker} \delta_{V}^{2}$ and the "coboundaries" are the elements of $\operatorname{im}_{V}^{1}$. Therefore, the $\mathfrak{e}$ of the long sequence (2) is identical with $\mathfrak{C}^{1}$ here. Moreover, as observed by Harrison, $\mathcal{E}^{2}(A, M)$ is the Baer group of equivalence classes of extensions $0 \rightarrow M \rightarrow B \rightarrow A \rightarrow 0$ (where $B$ is a commutative algebra), and similarly for $A / \mathfrak{a}$. Thus, the terms in the present long sequence coincide with those in the sequence (2) up to and including $\mathfrak{C}^{1}$. Now if $A$ is a polynomial algebra, possibly in infinitely many variables, then Harrison's $\mathcal{E}^{3}(A, M)$ vanishes by Theorem 11 of [5], while the sequence (1) represents zero because there is a splitting map $A \rightarrow B$. Since, likewise, $\mathcal{E}^{2}(A, M)=0$, it follows that for such an $A$ the two definitions of $\mathcal{E}^{3}(A / \mathfrak{a}, M)$ both coincide with $\mathfrak{e}^{1}$. Since every commutative algebra is a quotient of a polynomial algebra, we have proven finally the

THEOREM. For the category of commutative algebras over a field $k$, the third cohomology module $\mathcal{E}^{3}(A, M)$ of an algebra $A$ with coefficients in a module $M$ as defined in [4] coincides with the module $\varepsilon^{3}(A, M)$ of Harrison [5].

The long exact sequence analogous to (4) for associative algebras and groups has been studied by Barr and Rinehart [2].

\section{REFERENCES}

1. M. Barr, On the commutative cohomology theory, Bull. Amer. Math. Soc. (toappear).

2. M. Barr, and Rinehart, Cohomology as the derived functor of derivations, Trans. Amer. Math. Soc. 122 (1966), 416-426.

3. S. Eilenberg and S. Mac Lane, On the groups $H(\pi, n)$. I, Ann. of Math. (2) 58 (1953), 55-106.

4. M. Gerstenhaber, On the deformation of rings and algebras. II, Ann. of Math. (2) 84 (1966), 1-19.

5. D. K. Harrison, Commutative algebras and cohomology, Trans. Amer. Math. Soc. 104 (1962), 191-204.

6. G. Hochschild, On the cohomology theory for associative algebras, Ann. of Math. (2) 47 (1946), 568-579.

University of Pennsyluania 